\title{
Visual Modulation of Resting State $\alpha$ Oscillations
}

\author{
(D) Kelly Webster ${ }^{1}$ and 1 Tony Ro ${ }^{1,2,3}$
}

https://doi.org/10.1523/ENEURO.0268-19.2019

${ }^{1}$ Program in Psychology, ${ }^{2}$ Program in Biology, and ${ }^{3}$ Program in Cognitive Neuroscience, The Graduate Center of the City University of New York, New York, NY 10016

\begin{abstract}
Once thought to simply reflect passive cortical idling, recent studies have demonstrated that $\alpha$ oscillations play a causal role in cognition and perception. However, whether and how cognitive or sensory processes modulate various components of the $\alpha$ rhythm is poorly understood. Sensory input and resting states were manipulated in human subjects while electroencephalography (EEG) activity was recorded in three conditions: eyes-open fixating on a visual stimulus, eyes-open without visual input (darkness), and eyes-closed without visual input (darkness). We show that $\alpha$ power and peak frequency increase when visual input is reduced compared to the eyes open, fixating condition. These results suggest that increases in $\alpha$ power reflect a shift from an exteroceptive to interoceptive state and that increases in peak frequency following restricted visual input (darkness) may reflect increased sampling of the external environment in order to detect stimuli. They further demonstrate how sensory information modulates $\alpha$ and the importance of selecting an appropriate resting condition in studies of $\alpha$.
\end{abstract}

Key words: $\alpha$ peak frequency; $\alpha$ power; EEG; neural oscillations

\section{Significance Statement}

$\alpha$ oscillations have long been considered to reflect a stable neural trait, but we demonstrate that $\alpha$ varies with sensory input. By manipulating both eye state and sensory input, we demonstrate that under resting state conditions, visual input drives changes in $\alpha$ power and peak frequency. These changes likely allow the visual system to dynamically switch between interoceptive and exteroceptive states to prioritize detection of weak and/or infrequent visual stimuli. This work has important implications for studies of resting state brain dynamics, which have traditionally employed a variety of resting state conditions that may or may not match task conditions in terms of sensory input.

\section{Introduction}

Neuronal oscillations have provided considerable insights into the dynamics of information processing and

Received July 10, 2019; accepted November 15, 2019; First published December 13, 2019.

The authors declare no competing financial interests.

Author contributions: K.W. performed research; K.W. analyzed data; K.W. and T.R. wrote the paper; T.R. designed research.

This work was supported by the National Science Foundation Behavioral and Cognitive Sciences Grant 1561518 (to T.R.).

Acknowledgements: We thank Jeremy D. Fesi for assistance in collecting the data for this study.

Correspondence should be addressed to Tony Ro at tro@gc.cuny.edu. https://doi.org/10.1523/ENEURO.0268-19.2019

Copyright $@ 2020$ Webster and Ro

This is an open-access article distributed under the terms of the Creative Commons Attribution 4.0 International license, which permits unrestricted use, cognition in the brain. Of particular interest in the fields of attention and perception is the $7-$ to $13-\mathrm{Hz} \alpha$ oscillation. Once thought to reflect cortical idling (Adrian and Matthews, 1934), the $\alpha$ rhythm is now known to play an active role in cognitive processing. Specifically, $\alpha$ is thought to be a mechanism of functional inhibition, in which $\alpha$ synchronization increases over task-irrelevant areas and decreases over task-relevant ones. Such changes may increase the signal-to-noise ratio in neural processing and improve performance. Indeed, enhanced visual perception and detection are associated with reduced $\alpha$ power (Ergenoglu et al., 2004; Hanslmayr et al., 2007; Romei et al., 2008; Mathewson et al., 2009) and certain phases of the $\alpha$ cycle (Mathewson et al., 2009; Jaegle and Ro,

distribution and reproduction in any medium provided that the original work is properly attributed. 
2014). The causal role of the $\alpha$ rhythm in subsequent perception and cognition has been well demonstrated (Thut et al., 2011; Jaegle and Ro, 2014), but the role of cognitive and sensory processes in modulation of the $\alpha$ rhythm is unclear.

Task-related changes in $\alpha$ power may reflect changes in attentional direction or cognitive state (Worden et al., 2000). Ray and Cole (1985a,b) demonstrated that $\alpha$ power increases over parietal electrodes during tasks that induce an interoceptive state (i.e., attention toward internal cognitive processes) relative to those that induce an exteroceptive state (i.e., attention toward external input). Similarly, $\alpha$ power increases during mental imagery compared to actual perception (Schupp et al., 1994; Cooper et al., 2003). However, changes in $\alpha$ power have been demonstrated in contexts where the role of attention is less clear, like during eye closure (Berger, 1929). Therefore, there may be different mechanisms for these power changes.

Studies have attempted to elucidate whether $\alpha$ power changes during eye closure are endogenously or exogenously driven. Adrian and Matthews (1934) demonstrated that while the $\alpha$ rhythm is induced most readily during eye closure, any uniform visual field can evoke $\alpha$, suggesting an attentionally-driven effect. More recently, Boytsova and Danko (2010) demonstrated that eye closure modulates $\alpha$ power in darkness, and Ben-Simon et al. (2013) extended these findings to light conditions. Interestingly, comparing eyes-open conditions indicated that occipital $\alpha$ power increases in darkness compared to light. While this suggests that sensory input affects $\alpha$ power over posterior regions, Ben-Simon et al. (2013) focused the remainder of their interpretations on the frontal $\alpha$ effect and concluded that shifts in attentional direction modulate changes in $\alpha$ power. Therefore, the magnitude of sensory and state change effects on $\alpha$ power and the role of attention remain unclear.

The effects of endogenous and exogenous changes on other attributes of the $\alpha$ rhythm, like peak frequency, are even less understood. $\alpha$ peak frequency has long been considered to be a stable trait with high test-retest reliability (Gasser et al., 1985; Salinsky et al., 1991; Kondacs and Szabó, 1999; Grandy et al., 2013). However, recent evidence suggests that changes in cognitive (Haegens et al., 2014) and physical (Hülsdünker et al., 2016) demands cause shifts in $\alpha$ peak frequency.

The role of $\alpha$ peak frequency on subsequent perception has received considerable interest (Cecere et al., 2015; Samaha and Postle, 2015; Ro, 2019), and recently, studies have begun to investigate the opposite question: how sensory changes influence the $\alpha$ rhythm. However, these sensory effects are unclear and may be influenced by task and/or stimulus parameters. For example, some studies indicate that under task conditions, $\alpha$ peak frequency increases with increasing luminance of a peripheral stimulus (Cohen, 2014), while others indicate that peak frequency decreases or stays the same (Benedetto et al., 2018). Conversely, under resting conditions, $\alpha$ power, but not peak frequency, increases with reduced luminance (Benedetto et al., 2018). Neither study investigated con- ditions of complete darkness or examined the effect of eye closure. Therefore, whether and how sensory input affects the $\alpha$ rhythm independent of eye closure remains inconclusive.

To clarify how the $\alpha$ rhythm is modulated, we measured $\alpha$ power and peak frequency while manipulating eye state and sensory input. If $\alpha$ is modulated by endogenous changes, like attentional direction, power and peak frequency differences would be expected between eyesopen and eyes-closed conditions, regardless of sensory input. However, if $\alpha$ is modulated by sensory input, power and peak frequency differences would be expected as visual input is eliminated, such as when the eyes are closed or the lights are extinguished. Understanding how the $\alpha$ rhythm is modulated speaks to a broader question of what $\alpha$ represents and how $\alpha$ changes facilitate cognitive and sensory functioning.

\section{Materials and Methods}

This research was approved by the Institutional Review Board of the City University of New York. All participants gave written informed consent before participation.

\section{Participants}

Eighteen adults were recruited for participation in this study. Of these, two were excluded from the analyses: one because she did not complete all three experimental conditions, and one because he reported seeing some light during the eyes-open dark (EOD) condition. The remaining 16 participants (five females, mean age of 28.06 years, $21-40$ years old) completed all three conditions.

\section{Electroencephalography (EEG) recording and analysis}

EEG activity was recorded from 18 gold electrodes using Grass amplifiers (Natus Medical Inc.) at a $1000-\mathrm{Hz}$ sampling rate with an online bandpass filter of $0.1-100$ $\mathrm{Hz}$. Scalp electrodes were placed at the following locations in the 10-20 system: F3, Fz, F4, C3, Cz, C4, P3, Pz, $\mathrm{P} 4, \mathrm{O} 1, \mathrm{Oz}, \mathrm{O} 2$. Electrodes were also placed over the right and left mastoids, above and below the left eye, and lateral to the outer canthus of the right eye. The ground electrode was placed on the forehead. EEG activity was referenced online to the left mastoid and re-referenced offline to the average of the two mastoids. In each condition, data were recorded continuously for $\sim 5.5 \mathrm{~min}$ to allow for high-resolution frequency analyses of the data.

The EEG data were analyzed in $\mathrm{R}$ with minimal preprocessing to avoid distortion of the data. Electrooculographic (EOG) artifacts originating from blinks and eye movements were removed from the raw data using ICA with the infomax ICA algorithm (Bell and Sejnowski, 1995). The independent components were visually inspected by the experimenter after the data were coded blind to condition to eliminate any potential biases. EOG components were identified through inspection of component activity, scalp maps, and power-frequency spectra. For each subject and condition, one component representing blink activity and one component representing horizontal (i.e., saccadic) activity was removed. Data were inspected af- 
ter ICA removal to confirm that EOG artifacts were successfully removed. A sliding temporal window was used to epoch the data from each condition into 10-s segments with $90 \%$ overlap, resulting in 322 epochs. This epoching approach improved the reliability of the spectral estimates while maintaining high-frequency resolution. For each epoch, power spectra were computed using a fast Fourier transform (FFT) over each 10-s segment and were log transformed to reduce the $1 / f$ effect. To analyze the overall effect of resting state condition on $\alpha$ peak frequency and power, the FFTs over each epoch for each condition were averaged. $\alpha$ power was defined as the maximum peak deflection within the $7-$ to $13-\mathrm{Hz}$ window; the main effects of resting state condition and electrode on $\alpha$ power were replicated when using the mean power across the $\alpha$ window rather than the maximum peak inflection. An $\alpha$ peak could be identified for all subjects in all channels and conditions. Peak alpha frequencies for each subject in each condition can be seen in Figure 1. Because we were able to identify a peak within the $\alpha$ range for all subjects, $\alpha$ peak frequency was defined as the frequency of the maximum deflection within the 7- to $13-\mathrm{Hz}$ window. This minimized the potential confounding effects of power differences on frequency extraction with a center-of-mass analysis.

\section{Procedure}

EEG activity was recorded during three within-subjects resting conditions that varied in visual input and eye state. In the eyes-open fixation (EOF) condition, participants were instructed to fixate on the center of a screen flanked by four black fixation crosses (each subtending $0.2^{\circ}$ by $0.2^{\circ}$ visual angle for the horizontal and vertical components, respectively) on a gray background (luminance $=$ $14.18 \mathrm{~cd} / \mathrm{m}^{2}$ ). In the eyes-open dark (EOD) and eyesclosed dark (ECD) conditions, all light sources in the room were eliminated and the participant wore opaque goggles to further eliminate any light from entering the eyes. In the EOD condition, participants had their eyes open, whereas in the ECD condition, participants were instructed to close their eyes. In both dark conditions, participants were instructed to keep their eyes still, as though they were looking at an invisible fixation point at the center of their vision. Participants did not undergo dark adaptation before recording the dark conditions. EOG activity was used to confirm that participants complied with the instructions (e.g., blinks occurred during the EOF and EOD conditions and did not occur during the ECD condition and eye movements were equally minimized in all three conditions). The order of the conditions was counterbalanced across participants.

\section{Statistical analysis}

Data were analyzed using paired $t$ tests, two-way repeated measures (RM) ANOVA, and three-way RM ANOVA using R. Post hoc analyses for two-way and three-way RM ANOVA were performed using planned paired $t$ tests and $t$ tests corrected with false discover rate (FDR) for multiple comparisons. Significant differences were accepted at $p<0.05$. For a summary of the statistical tests, see Table 1.

\section{Results}

\section{Eye movements}

Eye movements and blinks were measured and analyzed before their removal with ICA to ensure that participants complied with instructions. Frequent blinking during the ECD condition, for example, would suggest that participants had their eyes open, obscuring differences in $\alpha$ between eyes-open and closed states. Significant reductions in eye movements in the EOF condition compared to the ECD and EOD conditions could suggest that more attentional resources or cognitive control were employed to maintain fixation in the EOF condition, which would likely affect $\alpha$ independently of any influences of sensory input.

To compare rates of blinking across the three conditions, we counted the number of blinks occurring in each 25.5-min recording session before removal using ICA. These blink data were submitted to a RM ANOVA with resting state condition as a within-subjects factor and subject as a random-effects factor. There was a significant difference in the rate of blinking across the three conditions, $F_{(2,30)}=14.80, p<0.001, \eta_{\mathrm{p}}{ }^{2}=0.50^{\mathrm{a}}$. The significant $F$ test was followed up by planned pairwise comparisons. Blinking was significantly reduced in the $\mathrm{ECD}$ (mean $=4.19, \mathrm{SD}=5.47$ ) condition compared to the EOD (mean $=36.26, \mathrm{SD}=38.26 ; t_{(15)}=3.36, p=0.004$, $d=0.84^{\mathrm{b}}$ ) and EOF (mean $=70.5$, SD $=52.31 ; t_{(15)}=$ 5.09, $p<0.001, d=1.27^{\mathrm{b}}$ ) conditions, confirming that participants complied with the instructions to close their eyes in the ECD condition. Blinking was also reduced in the EOD condition compared to the EOF condition, $t_{(15)}=$ $2.54, p=0.02, d=0.63^{\mathrm{b}}$.

To compare rates of eye movements across the three conditions, we counted the number of eye movements occurring in each $\sim 5.5$ min session before removal using ICA. These eye movement data were submitted to a RM ANOVA with resting state condition as a within-subjects factor and subject as a random-effects factor. There was no significant difference in the rate of eye movements across the three conditions, $F_{(2,30)}=0.38, p=0.69, \eta_{p}^{2}=$ $0.02^{c}$. This suggests that eye movements were equally minimized across all three groups and that any differences in $\alpha$ across the three conditions cannot be explained by differences in attention or cognitive control exerted to maintain fixation.

\section{$\alpha$ peak frequency}

$\alpha$ peak frequency was measured over the 12 scalp electrodes in each of the three resting state conditions (Fig. 2A). Before analysis of peak frequency across resting state conditions, the data were first investigated for internal consistency. This was achieved by comparing the peak $\alpha$ frequency data from the first half of the recording session to the second half of the recording session to measure split-half reliability. Peak frequency data were averaged across electrodes for each subject in each condition and recording half. There was high internal consistency across all three resting state conditions $\left[r_{\mathrm{EOF}}(14)=\right.$ $0.91, r_{\mathrm{EOD}}(14)=0.98, r_{\mathrm{ECD}}(14)=0.90$; correlation coefficients are corrected by the Spearman-Brown formula for 

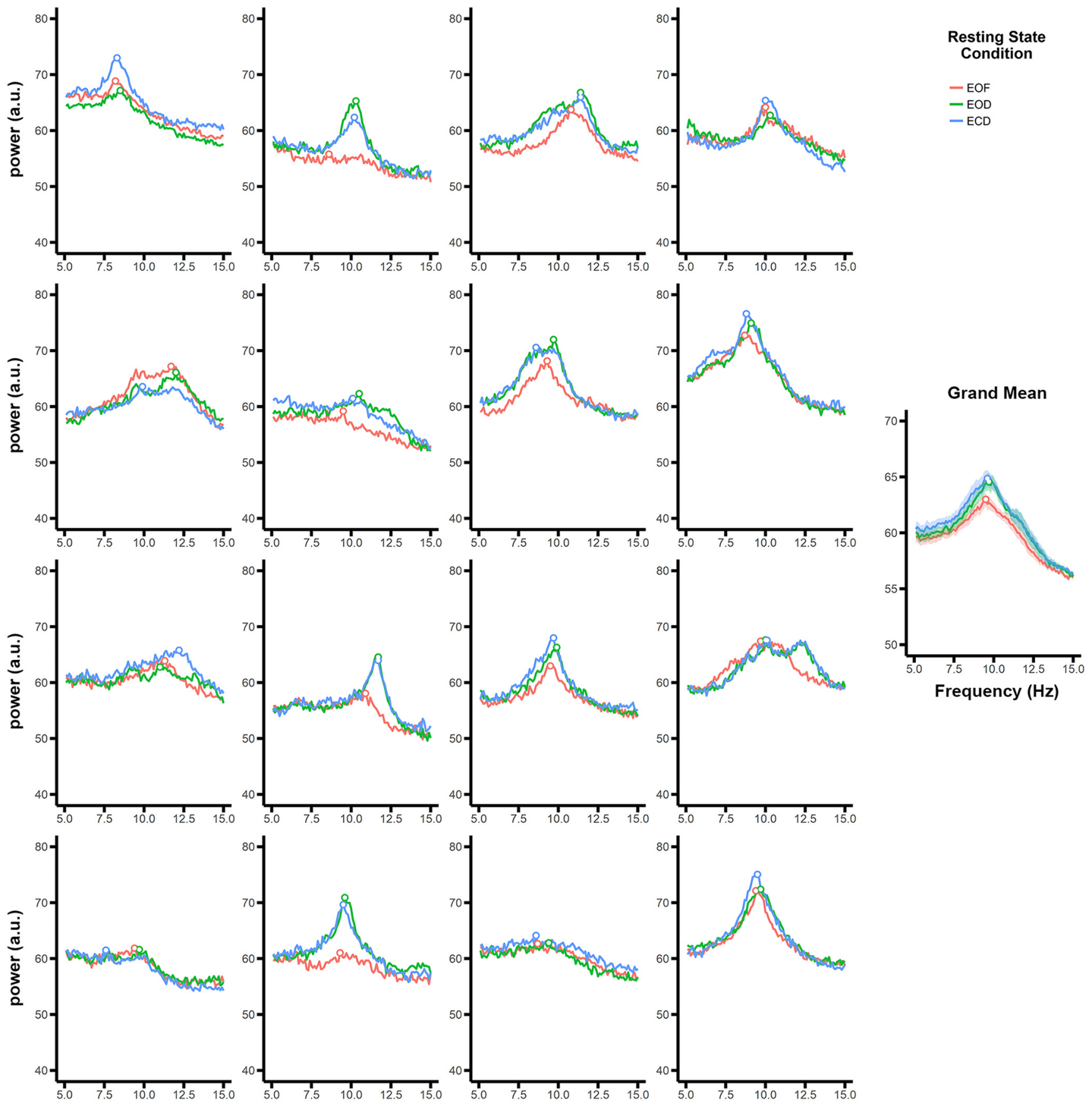

Frequency $(\mathrm{Hz})$

Figure 1. Power spectra for each subject in each of the conditions. Each panel represents data from one subject averaged across electrodes. Points represent the peak frequency for each condition. The grand mean across subjects is shown on the right. Shaded regions represent the within-subject SE (Cousineau, 2005; Morey, 2008).

split-half reliability; Stanley, 1971], suggesting that our measure of peak $\alpha$ frequency was reliable and consistent across the recording sessions. Following analysis of internal consistency, the peak frequency data were submitted to an omnibus ANOVA with resting state condition and electrode site as within-subjects factors and subjects as a random-effects factor to compare the effects of eye closure and visual modulation on $\alpha$ peak frequency. There were significant main effects of resting state condition
$\left(F_{(2,30)}=10.26, p<0.001, \eta_{\mathrm{p}}{ }^{2}=0.41^{\mathrm{d}}\right)$ and electrode site $\left(F_{(11,165)}=8.11, p<0.001, \eta_{\mathrm{p}}{ }^{2}=0.35^{\mathrm{e}}\right)$ on $\alpha$ peak frequency. There was no significant interaction between resting state condition and electrode site $\left(F_{(22,330)}=1.13\right.$, $p=0.32, \eta_{\mathrm{p}}^{2}=0.07^{f}$.

The significant main effects were followed up by planned paired comparisons. $\alpha$ peak frequency was significantly reduced in the EOF condition (mean $=9.46 \mathrm{~Hz}$, $\mathrm{SD}=0.81 \mathrm{~Hz}$ ) compared to the EOD condition (mean = 
Table 1. Statistical table

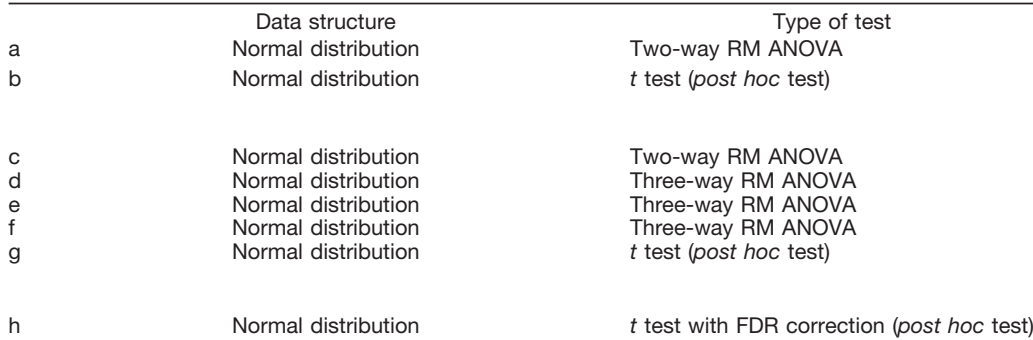

$\eta_{\mathrm{p}}^{2}=0.50$

ECD vs. EOD, mean $=-31.44(-51.38$ to -11.50$)$ $E C D$ vs. EOF, mean $=-66.31(-94.07$ to -38.56$)$ EOD vs. EOF, mean $=-34.88(-64.18$ to -5.57$)$

$\eta_{p_{2}}^{2}=0.02$

$\eta_{p_{2}}=0.41$

$\eta_{p_{2}}=0.35$

$\eta_{\mathrm{p}}^{2}=0.07$

EOF vs. EOD, mean $=-.59(-1.01$ to -.17$)$

EOF vs. ECD, mean $=-.74(-1.12$ to -.37$)$

$E O D$ vs. $E C D$, mean $=-.16(-.30$ to .12$)$

$\mathrm{P} 3$ vs. $\mathrm{F} 3$, mean $=.71(.25$ to 1.17$)$

$\mathrm{P} 3$ vs. $\mathrm{Fz}$, mean $=.67$ (.26 to 1.08$)$

$\mathrm{P} 3$ vs. F4, mean $=.63(.24$ to 1.03$)$

$\mathrm{P} 3$ vs. $\mathrm{C} 3$, mean $=.43(.11$ to .75$)$

$\mathrm{P} 3$ vs. $\mathrm{Cz}$, mean $=.54$ (.13 to .94$)$

$\mathrm{P} 3$ vs. C4, mean $=.63(.28$ to .99$)$

$\mathrm{Pz}$ vs. $\mathrm{F} 3$, mean $=.76$ (.26 to 1.26$)$

$\mathrm{Pz}$ vs. Fz, mean $=.72$ (.33 to 1.11$)$

$\mathrm{Pz}$ vs. $\mathrm{F} 4$, mean $=.68$ (.29 to 1.07$)$

$\mathrm{Pz}$ vs. C3, mean $=.48(.13$ to .83$)$

Pz vs. $\mathrm{Cz}$, mean $=.59(.20$ to .97$)$

$\mathrm{Pz}$ vs. $\mathrm{C} 4$, mean $=.68$ (.30 to 1.07$)$

$\mathrm{P} 4$ vs. $\mathrm{F} 3$, mean $=.66$ (.16 to 1.17$)$

$\mathrm{P} 4$ vs. Fz, mean $=.62(.21$ to 1.04$)$

P4 vs. F4, mean $=.59(.19$ to .99$)$

$\mathrm{P} 4$ vs. $\mathrm{C} 3$, mean $=.39(-.003$ to .78$)$

$\mathrm{P} 4$ vs. $\mathrm{Cz}$, mean $=.49$ (.05 to .93$)$

$\mathrm{P} 4$ vs. $\mathrm{C} 4$, mean $=.59(.27$ to .90$)$

01 vs. F3, mean $=.81(.34$ to 1.29$)$

O1 vs. Fz, mean $=.77$ (.30 to 1.23 )

01 vs. F4, mean $=.73(.27$ to 1.19$)$

01 vs. $\mathrm{C} 3$, mean $=.53(.14$ to .91$)$

01 vs. $\mathrm{Cz}$, mean $=.63(.17$ to 1.09$)$

$\mathrm{O} 1$ vs. $\mathrm{C} 4$, mean $=.73(.32$ to 1.14$)$

$\mathrm{Oz}$ vs. $\mathrm{F} 3$, mean $=.73(.28$ to 1.17$)$

Oz vs. Fz, mean $=.69$ (.24 to 1.13$)$

Oz vs. F4, mean $=.65(.19$ to 1.10$)$

$\mathrm{Oz}$ vs. C3, mean $=.45$ (.07 to .82)

$\mathrm{Oz}$ vs. $\mathrm{Cz}$, mean $=.55$ (.08 to 1.02$)$

$\mathrm{Oz}$ vs. $\mathrm{C} 4$, mean $=.65$ (.24 to 1.06$)$

$\mathrm{O} 2$ vs. $\mathrm{F} 3$, mean $=.74(.25$ to 1.24$)$

$\mathrm{O} 2$ vs. Fz, mean $=.70$ (.24 to 1.16$)$

$\mathrm{O} 2$ vs. $\mathrm{F} 4$, mean $=.66(.19$ to 1.14$)$

$\mathrm{O} 2$ vs. $\mathrm{C} 3$, mean $=.46$ (.08 to .85$)$

$\mathrm{O} 2$ vs. $\mathrm{Cz}$, mean $=.57$ (.09 to 1.04$)$

$\mathrm{O} 2$ vs. $\mathrm{C} 4$, mean $=.66$ (.24 to 1.09$)$

$\mathrm{F} 3$ vs. Fz, mean $=-.04(-.29$ to .21$)$

$\mathrm{F} 3$ vs. $\mathrm{F} 4$, mean $=-.08(-.39$ to .23$)$

$\mathrm{F} 3$ vs. $\mathrm{C} 3$, mean $=-.28(-.58$ to .03$)$

$\mathrm{F} 3$ vs. $\mathrm{Cz}$, mean $=-.17(-.47$ to .12$)$

$\mathrm{F} 3$ vs. $\mathrm{C} 4$, mean $=-.08(-.52$ to .37$)$

Fz vs. F4, mean $=-.04(-.19$ to .11$)$

Fz vs. C3, mean $=-.24(-.54$ to .07$)$

Fz vs. Cz, mean $=-.14(-.29$ to .02$)$

Fz vs. C4, mean $=-.04$ (-.41 to .34$)$

$\mathrm{F} 4$ vs. $\mathrm{C} 3$, mean $=-.20(-.53$ to .13$)$

$\mathrm{F} 4$ vs. $\mathrm{Cz}$, mean $=-.10(-.27$ to .07$)$

$\mathrm{F} 4$ vs. $\mathrm{C} 4$, mean $=0.00(-.29$ to .29$)$

$\mathrm{C} 3$ vs. $\mathrm{Cz}$, mean $=.10(-.14$ to .34$)$

$\mathrm{C} 3$ vs. $\mathrm{C} 4$, mean $=.20(-.18$ to .58$)$

$\mathrm{Cz}$ vs. $\mathrm{C} 4$, mean $=.10(-.29$ to .48$)$

$\mathrm{P} 3$ vs. $\mathrm{Pz}$, mean $=-.05$ ( -.17 to 07$)$

$\mathrm{P} 3$ vs. $\mathrm{P} 4$, mean $=.05(-.11$ to .20$)$

$\mathrm{P} 3$ vs. 01 , mean $=-.10(-.24$ to .04$)$

$\mathrm{P} 3$ vs. Oz, mean $=-.01(-.18$ to .15$)$

$\mathrm{P} 3$ vs. $\mathrm{O} 2$, mean $=-.03(-.18$ to .12$)$

$\mathrm{Pz}$ vs. $\mathrm{P} 4$, mean $=.10(-.07$ to .26$)$

$\mathrm{Pz}$ vs. 01 , mean $=-.05(-.26$ to .17$)$

$\mathrm{Pz}$ vs. Oz mean $=.04(-.21$ to .28$)$

$\mathrm{Pz}$ vs. $\mathrm{O} 2$, mean $=.02(-.19$ to .23$)$

$\mathrm{P} 4$ vs. 01 , mean $=-.14(-.37$ to .09$)$

$\mathrm{P} 4$ vs. $\mathrm{Oz}$, mean $=-.06(-.28$ to .16$)$

$\mathrm{P} 4$ vs. 02 , mean $=-.08(-.27$ to .11$)$

01 vs. $\mathrm{Oz}$, mean $=.08(-.05$ to .21$)$

01 vs. 02 , mean $=.06(-.08$ to .21$)$

$\mathrm{Oz}$ vs. $\mathrm{O} 2$, mean $=-.01(-.12$ to .09$)$

$\eta_{p_{2}}^{2}=0.30$

$\eta_{p_{2}}=0.37$

EOF vs. EOD, mean $=-.02(-.05$ to .00$)$

EOF vs. ECD, mean $=-.03(-.05$ to -.01$)$

EOD vs. ECD, mean $=-.01(-.02$ to .01$)$ 
Table 1. Continued

\begin{tabular}{ccc}
\hline Data structure & Type of test \\
$\mathrm{m}$ & Normal distribution & $t$ test with FDR correction (post hoc test)
\end{tabular}

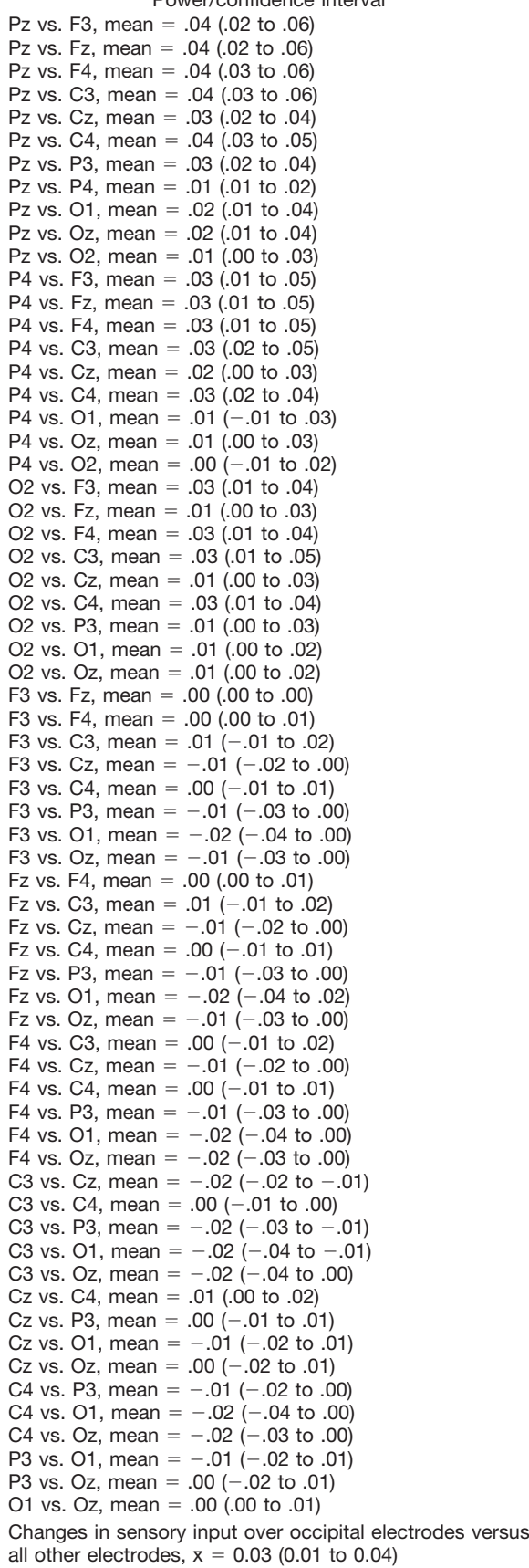

Table summarizes the distribution, statistical test, and power or confidence interval for each statistical test in the present study. Identifiers refer to superscript identifiers in the main text.

$10.05 \mathrm{~Hz}, \mathrm{SD}=0.96 \mathrm{~Hz} ; t_{(15)}=3.07, p=0.008, d=0.77^{9}$; all tests are two-tailed with $\alpha=0.05$ ) and the ECD condition $\left(\right.$ mean $=10.14 \mathrm{~Hz}, \mathrm{SD}=1.02 \mathrm{~Hz} ; t_{(15)}=3.72, p=$ $0.002, d=0.93^{9}$; Fig. 3). There were no significant differences between the EOD and ECD conditions $\left(t_{(15)}=0.92\right.$, $p=0.37, d=0.23^{9}$ ), demonstrating that the restriction of light produced these changes rather than the eyes being opened or closed.

As can be seen in Figure $2 B$, the significant effect of electrode site is driven by higher peak frequencies over posterior electrode sites compared to frontal electrode sites. Peak frequencies over posterior electrodes (P3, Pz, $\mathrm{P} 4, \mathrm{O} 1, \mathrm{Oz}$, and O2) were significantly higher than peak frequencies over frontal electrodes (F3, Fz, F4, C3, Cz, and C4; all $t s \geq 2.52$, all $p s \leq 0.046$, all $d s \geq 0.63^{\mathrm{h}}$, FDR corrected for multiple comparisons; with the exception of the comparisons between C3 and P4 $\left(t_{(15)}=2.12, p=\right.$ $\left.0.09, d=0.53^{\mathrm{h}}\right)$ and $\mathrm{Cz}$ and P4 $\left(t_{(15)}=2.36, p=0.06, d\right.$ $\left.=0.59^{\mathrm{h}}\right)$, which were marginally significant). There were no significant differences in peak frequency across frontal 
A
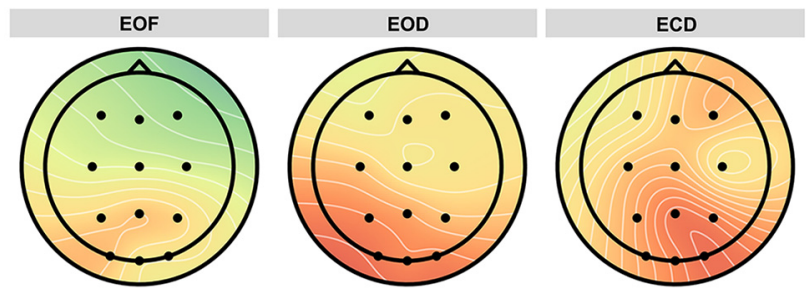

B

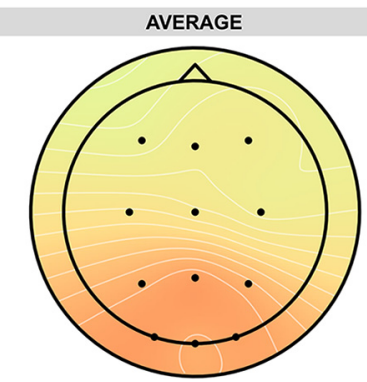

Peak Alpha

Frequency

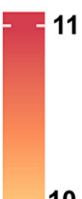

10

9

$-8$

Figure 2. Distribution of $\alpha$ peak frequency across the scalp. $\boldsymbol{A}, \alpha$ peak frequency in each resting state condition. $\boldsymbol{B}, \alpha$ peak frequency averaged across resting state conditions.

electrodes (all $t s \leq 1.93$, all $p s \geq 0.13$, all $d s \leq 0.48^{\mathrm{h}}$, FDR corrected for multiple comparisons) or across posterior electrodes (all $t s \leq 1.46$, all $p s \geq 0.27$, all $d s \leq 0.36^{\mathrm{h}}$, FDR corrected for multiple comparisons).

\section{$\alpha$ power}

$\alpha$ power at the $\alpha$ peak frequency was measured over the 12 scalp electrodes in each of the three resting state conditions. Within each subject, power values were normalized by dividing individual power values for each electrode by the mean power across all electrodes and conditions. The spatial distribution of $\alpha$ power across resting state conditions is shown in Figure $4 A$. Before analysis of $\alpha$ power across resting state conditions, the data were first investigated for internal consistency. This was achieved by comparing the $\alpha$ power data from the first half of the recording session to the second half of the recording session to measure split-half reliability. Power data were averaged across electrodes for each subject in each condition and recording half. There was high internal consistency across all three resting state conditions $\left[r_{\mathrm{EOF}}(14)=0.97, r_{\mathrm{EOD}}(14)=0.95, r_{\mathrm{ECD}}(14)=0.96\right.$; correlation coefficients are corrected by the Spearman-Brown formula for split-half reliability; Stanley, 1971], suggesting that our measure of $\alpha$ power was reliable and consistent across the recording sessions. Following analysis of internal consistency, normalized power values were submitted to an omnibus ANOVA with resting state condition and electrode as within-subjects factors and subjects as a random-effects factor. There were significant main effects of resting state condition $\left(F_{(2,30)}=6.51, p=0.004, \eta_{p}^{2}=\right.$ $\left.0.30^{i}\right)$ and electrode site $\left(F_{(11,165)}=8.82, p<0.001, \eta_{p}^{2}=\right.$ $\left.0.37^{j}\right)$ on normalized $\alpha$ power. There was also a significant interaction between resting state condition and electrode site $\left(F_{(22,330)}=1.84, p=0.01, \eta_{p}^{2}=0.10^{k}\right)$.

The significant main effects and interactions were followed up by planned paired comparisons. $\alpha$ power was significantly reduced in the EOF condition (mean $=0.98$, $\mathrm{SD}=0.03$ ) compared to the EOD (mean $=1.01, \mathrm{SD}=$ $\left.0.03 ; t_{(15)}=2.32, p=0.03, d=0.58^{\prime}\right)$ and ECD conditions

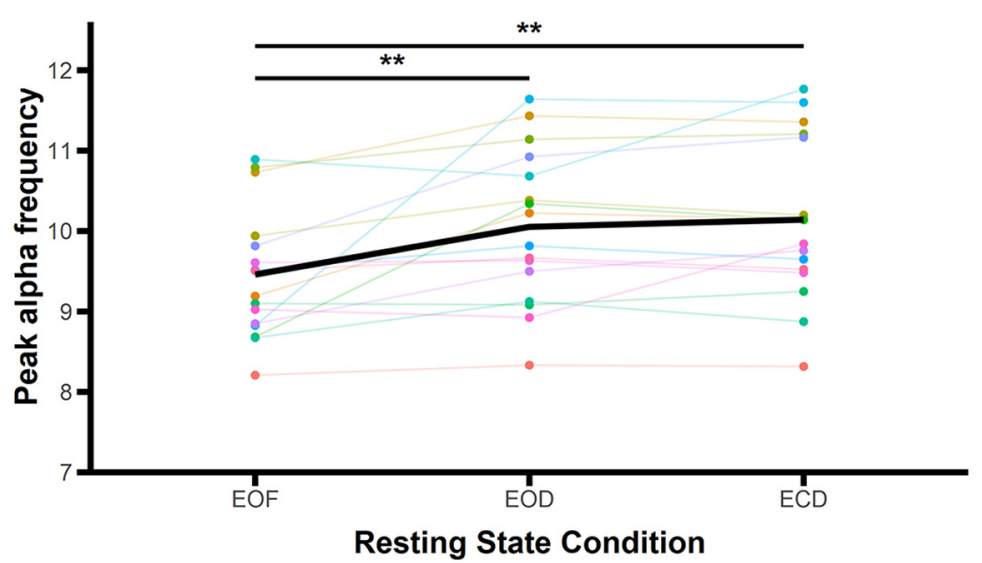

Figure 3. Mean $\alpha$ peak frequency across resting state conditions. Colored points and lines indicate data from individual subjects. The bold black line represents the mean averaged across subjects; $* * p<0.01$. 
A

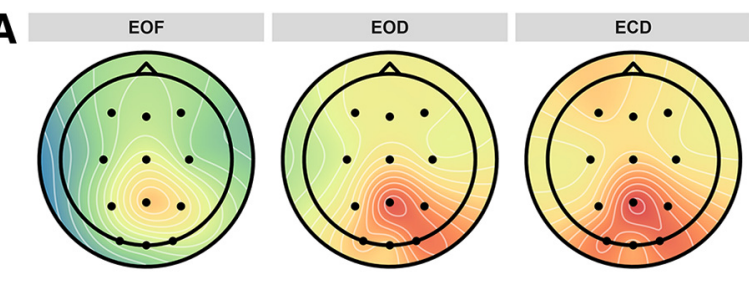

B

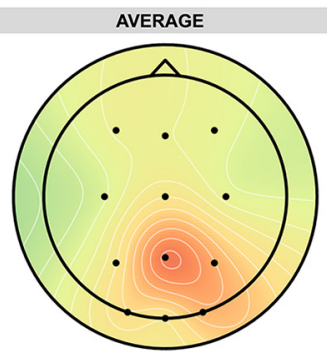

Percent change in power from mean

$-4 \%$

$2 \%$

$0 \%$

$-2 \%$

$-4 \%$

Figure 4. Distribution of normalized $\alpha$ power across the scalp. Power is plotted as percentage change from the mean across all resting state conditions, electrodes, and subjects to better illustrate the differences in power occurring across different resting state conditions. Power values that deviate strongly from the grand mean will be non-zero in the averaged data. $\boldsymbol{A}$, Normalized $\alpha$ power in each resting state condition. $\boldsymbol{B}$, Normalized $\alpha$ power averaged across resting state conditions.

$\left(\right.$ mean $=1.01, \mathrm{SD}=0.02 ; t_{(15)}=3.38, p=0.004, d=$ 0.85 ; Fig. 5). There was no significant difference between EOD and ECD conditions $\left(t_{(15)}=0.75, p=0.47, d=\right.$ $\left.0.19^{\prime}\right)$, suggesting that changes in $\alpha$ power, like frequency, were modulated by sensory input, not eye closure.

As can be seen in Figure $4 B$, the significant effect of electrode site is largely driven by higher power over the posterior electrodes, in particular, $\mathrm{Pz}, \mathrm{P} 4$, and $\mathrm{O} 2$, compared to other electrodes. $\alpha$ power was significantly higher in electrode $\mathrm{Pz}$ compared to all other electrodes except for $\mathrm{O} 2$ (all $t \mathrm{~s} \geq 3.04$, all $p s \leq 0.027$, all $d s \geq 0.76^{\mathrm{m}}$, FDR corrected for multiple comparisons). Additionally, power over $\mathrm{P} 4$ and $\mathrm{O} 2$ was significantly higher than over most of the frontal electrodes (F3, Fz, F4, C3, and C4; power over $\mathrm{O} 2$ was also significantly higher than electrode $\mathrm{Oz}$ and power over $\mathrm{P} 4$ was also significantly higher than electrode P3, all $t s \geq 2.73$, all $p s \leq 0.04$, all $d s \geq$ $0.68^{\mathrm{m}}$, FDR corrected for multiple comparisons). Power over several of the frontal electrodes was also significantly reduced relative to some of the other electrodes (power over F4 was significantly reduced compared to $\mathrm{Cz}\left(t_{\text {(df) }}=\right.$ $2.73, p=0.04, d=0.68^{\mathrm{m}}$, FDR corrected for multiple comparisons), power over $\mathrm{C} 3$ was significantly reduced compared to $\mathrm{Cz}, \mathrm{P} 3, \mathrm{O} 1$, and $\mathrm{Oz}$ (all $t \mathrm{~s} \geq 2.76$, all $p s \leq$ 0.04 , all $d s \geq 0.69^{m}$, FDR corrected for multiple comparisons), and power over C4 was significantly reduced compared to $\mathrm{Cz}\left(t_{(\mathrm{df})}=2.65, p=0.04, d=0.66^{\mathrm{m}}\right.$, FDR corrected for multiple comparisons).

To clarify the interaction between condition and electrode on $\alpha$ power, we examined how power changed across resting state conditions over each electrode. These changes are summarized in Table 2. As can be seen, restriction of visual input (EOD and ECD conditions compared to EOF condition) more strongly affects $\alpha$ power over occipital electrodes than over other electrodes. To confirm this, we tested differences in $\alpha$ power in light and dark conditions over occipital electrodes compared to the remaining electrodes. First, power in the dark

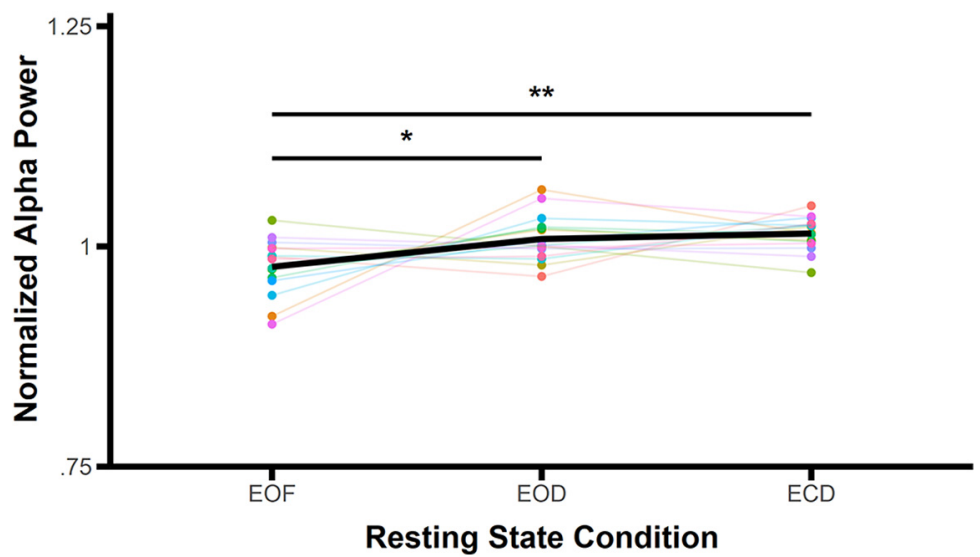

Figure 5. Mean normalized $\alpha$ power across resting state conditions. Colored points and lines indicate data from individual subjects; the same colors represent the same subjects as Figure 3 . The bold black line represents the mean averaged across subjects; $* p<$ $0.05, * * p<0.01$. 
Table 2. Percent changes in power across resting state conditions

\begin{tabular}{llll}
\hline Electrode & EOD-EOF & ECD-EOF & ECD-EOD \\
F3 & $2.62 \%$ & $3.65 \%$ & $1.05 \%$ \\
Fz & $2.30 \%$ & $3.28 \%$ & $1.00 \%$ \\
F4 & $2.78 \%$ & $3.49 \%$ & $0.72 \%$ \\
C3 & $2.28 \%$ & $2.95 \%$ & $0.69 \%$ \\
Cz & $1.98 \%$ & $2.79 \%$ & $0.83 \%$ \\
C4 & $2.43 \%$ & $3.19 \%$ & $0.78 \%$ \\
P3 & $2.77 \%$ & $3.25 \%$ & $0.50 \%$ \\
Pz & $3.20 \%$ & $3.57 \%$ & $0.39 \%$ \\
P4 & $2.88 \%$ & $3.26 \%$ & $0.39 \%$ \\
O1 & $4.96 \%$ & $5.58 \%$ & $0.66 \%$ \\
Oz & $4.15 \%$ & $4.56 \%$ & $0.43 \%$ \\
O2 & $5.17 \%$ & $5.07 \%$ & $-0.10 \%$ \\
\hline
\end{tabular}

conditions (EOD and ECD) were averaged per electrode per subject, as we found no power differences between these conditions. We subtracted the $\alpha$ power in the light condition (EOF) from the average dark condition per electrode per subject to get a difference score that represented changes in power as a result of light restriction. Then, for each subject, we averaged the occipital electrodes separately from the remaining electrodes. Changes in sensory input had a significantly greater effect on $\alpha$ power over occipital electrodes compared to the other electrodes, $t_{(15)}=2.87, p=0.01, d=0.72^{n}$.

The preceding analyses on the ICA-cleaned data and on data that had not been cleaned with ICA to remove eye movement and blink artifacts produced the same power and peak frequency results. Furthermore, data in the ECD condition were also analyzed after epochs with blinks artifacts were removed, which yielded the same power and frequency results. Together, these results suggest that eye movements and blinks did not significantly contaminate our data and that our ICA cleaning procedure did not distort or bias the results.

\section{Discussion}

We measured $\alpha$ peak frequency and power over three different resting state conditions that varied in visual input and found that visual input modulates both $\alpha$ peak frequency and power. Both peak frequency and power increased in the $\alpha$ band when visual input was restricted. This indicates that even under resting state conditions, $\alpha$ peak frequency and power are variable and highly dependent on visual input. Furthermore, we demonstrate that $\alpha$ peak frequency and power are significantly reduced in the presence of light, but that there are no differences between dark conditions with eyes open or closed. Thus, light input, not eye closure, modulates $\alpha$ peak frequency and power.

Interestingly, the finding that sensory input, not eye state, drives changes in the $\alpha$ rhythm is somewhat at odds with other results reported in the literature. For example, in a combined fMRI and EEG study by Ben-Simon et al. (2013), eye state and electrode location, but not light input, were found to have a significant effect on $\alpha$ amplitude. Similarly, a resting state fMRI study by Jao et al. (2013) demonstrated that eye closure, not light input, significantly modulates the strength of functional connec- tivity within the default mode network, BOLD signal variance, and fractional amplitude of low frequency fluctuation within sensory cortical areas, the thalamus, and insula. The authors of both studies conclude that the brain adopts distinctive configurations when the eyes are open versus closed that are independent of sensory input and may reflect endogenous changes between interoceptive and exteroceptive states.

Although the influences of eye state and light input on resting state dynamics were investigated in both of these studies, the methodology employed in the present study differed from that employed by Ben-Simon et al. (2013) and Jao et al. (2013) in a critical way. In the present study, we used one light condition, in which eyes were open, and two dark conditions, in which eyes were open and closed. However, both the Ben-Simon et al. (2013) and Jao et al. (2013) studies employed two light (eyes open and eyes closed) and two dark (eyes open and eyes closed) conditions. While the goal of this manipulation was to fully disentangle the effects of eye closure and light input on subsequent brain dynamics, it introduces a confound: closure of the eyelids likely changes rather than eliminates the amount of illumination reaching the eyes in the light condition. This effect will vary from individual to individual based on eyelid skin thickness and pigmentation. Therefore, when averaging across the eyes open and eyes closed conditions for each lighting condition, the light condition with eyes closed likely included some visual input, which was dependent on sample characteristics, such as skin thickness and tone.

In the present study, we did not measure the $\alpha$ rhythm under light, eyes closed conditions, but we were nonetheless able to interpret the effect of eye closure on the $\alpha$ rhythm by comparing the EOD and ECD conditions, as well as the effect of sensory input by comparing the eyes open light to both the EOD and ECD conditions. However, future studies that carefully control for both illumination (sensory input) and eye state may help to further elucidate the effects of these factors on resting state brain dynamics.

Also, unlike these previous studies, our results demonstrate for the first time that visual input modulates $\alpha$ peak frequency under resting conditions, and that these changes in $\alpha$ peak frequency directly mirror power changes. While $\alpha$ peak frequency is typically considered to be a stable trait variable (Gasser et al., 1985; Salinsky et al., 1991; Kondacs and Szabó, 1999; Grandy et al., 2013), more recent work has demonstrated that peak frequency also functions as a state variable, with fluctuations in peak frequency potentially reflecting changes in cognitive and/or physical engagement (Haegens et al., 2014; Gutmann et al., 2015). While a few other studies have begun to investigate the effects of visual input on $\alpha$ peak frequency, the results heretofore are largely mixed. For example, Cohen (2014) noted that $\alpha$ peak frequency increased with increasing luminance of a contralateral stimulus during an infrequent time-coordinated stimulus localization task. However, a study by Benedetto et al. (2018) found the opposite effect under task conditions: when the luminance of a covertly attended disk was 
altered, phase-locked $\alpha$ peak frequency increased in low ambient luminance conditions, whereas spontaneous $\alpha$ was unaffected. Furthermore, ambient luminance was found to have no effect on spontaneous $\alpha$ frequency under resting conditions. The differences in results between these two studies and the present one may be due to several factors. Differences in the task between the Cohen (2014) and Benedetto et al. (2018) studies, especially task difficulty and task-relevance of the luminancechanging stimulus, may account for the different effects of luminance on $\alpha$ frequency. Furthermore, only the luminance of a relatively small square stimulus was manipulated in the Cohen (2014) study, whereas ambient luminance was also manipulated in the Benedetto et al. (2018) study, as in the present study. While ambient luminance was manipulated in both the present study and in the Benedetto et al. (2018) study, Benedetto et al. recorded EEG in mesopic luminance following dark adaptation, whereas the present study did not require any period of dark adaptation and compared mesopic and scotopic conditions; the restricted luminance range used by Benedetto et al., may therefore have masked the differences observed in the present study. Nevertheless, a more thorough examination of the interplay between task demands, stimulus characteristics, and luminance is needed to clarify the effects of visual input on $\alpha$ peak frequency.

What do these newfound shifts in peak frequency and power with changes in sensory input represent? Haegens et al. (2014) demonstrated that individual $\alpha$ peak frequency increases with increasing task demands while $\alpha$ power decreases. The authors interpret this shift in frequency as reflecting either a reduction in the window of suppression, which may be beneficial with an increasingly difficult task, or activation of different neuronal populations with changing task demands. Here we demonstrate increases in both peak frequency and in power with changes in sensory input, although the "task" is unchanged across conditions. A similar underlying mechanism may account for increases in $\alpha$ peak frequency under both conditions of reduced visual input and increased task demands. In the Haegens et al. (2014) study, $\alpha$ peak frequency increased during 0-back and 2-back tasks relative to resting, baseline, and passive viewing conditions, suggesting that when a task is made more difficult, reducing the window of suppression may be beneficial to improving performance. Similarly, when visual input is extinguished, as in the present study, reducing the window of suppression allows the visual system to sample the external environment more frequently to detect visual stimuli. An alternative explanation is that when visual input is reduced, the brain shifts from an exteroceptive state to interoceptive state, which may be accompanied by activation of a different population of neurons whose activity may oscillate at a higher peak frequency.

Support for this latter explanation comes from the resting state $\mathrm{fMRI}$ literature. Recently, there has been a growing interest in the neural processing that occurs when we "do nothing," the so-called resting state. Eyes open and eyes closed states under varying degrees of visual stim- ulation have been interchangeably employed as resting conditions. Drawing from the EEG literature, however, there has been recent interest in whether these different resting conditions are actually equivalent, or if they produce distinct patterns of activity in the brain. Initially, studies determined through visual inspection that the default mode network was stable across different resting conditions (Greicius et al., 2003; Fox et al., 2005; Fransson, 2005). When compared statistically, however, studies have demonstrated that eyes open and eyes closed resting states produce different patterns of activity and connectivity in the brain. While there is some variability in the findings (possibly due to differences in data collection and processing), studies have generally demonstrated that BOLD activity is reduced under eyes open resting state conditions compared to eyes closed conditions (McAvoy et al., 2008; Bianciardi et al., 2009; Zou et al., 2009; Jao et al., 2013). Some studies have also reported decreases in functional connectivity under eyes open conditions relative to eyes closed conditions (Bianciardi et al., 2009; Zou et al., 2009; Xu et al., 2014; however, see Yan et al., 2009). Few studies have attempted to disentangle the effects of sensory stimulation from eye state on resting state activity, but one study by Jao et al. (2013) averaged across eyes open and eyes closed light conditions, which produce different illumination levels, impeding the interpretation of the effect of sensory input on subsequent activity as described above.

Different resting states have also been demonstrated to activate different systems within the brain, with eyes-open conditions producing activation in oculomotor and attentional systems and eyes closed conditions producing activation in sensory systems (Marx et al., 2003, 2004). These findings collectively suggest that eyes-open and eyes-closed resting states are two different states of cognition; eye closure induces an interoceptive state, characterized by imagination, planning, and multisensory activity, while eye opening induces an exteroceptive state characterized by attention, readiness, and oculomotor activity. The differences we see in $\alpha$ power and frequency in the present study may similarly reflect a state change from exteroception to interoception as visual input is restricted.

Interestingly, results from a recent study suggest that even within a single resting state condition, the brain does not remain tonically active in a single state of brain function (Fransson, 2005). Rather, there are low frequency $(0.012-0.1 \mathrm{~Hz})$ fluctuations in the BOLD signal that are synchronized across brain regions. Fransson (2005) demonstrated that these signal fluctuations were associated with changes in functional connectivity, such that the fluctuations in the BOLD signal represented the alternating activation of two distinct networks. This relationship between spontaneous low frequency BOLD fluctuations and functional connectivity was found in both eyes open and eyes closed resting states (although the two conditions were not compared statistically). Fransson speculated that during rest, the interoceptive state, which is characterized by inner thought and self-reflective thinking, is periodically interrupted as the brain shifts to an extero- 
ceptive state, which is characterized by increased readiness and attention to changes in the internal and external environment. These results are interesting in light of other studies that have suggested that eyes closed resting states activate networks consistent with an interoceptive state, while eyes open resting state activate networks consistent with an exteroceptive state. Together, these results might suggest that at rest, the brain shifts from an interoceptive to exteroceptive state routinely, with signal intensity and/or functional connectivity being stronger in the interoceptive network when the eyes are closed, and vice versa. However, additional research that statistically compares eyes open and eyes closed resting states, along with resting states that differ in sensory input, during these spontaneous low frequency fluctuations will be necessary to better understand these dynamic changes in brain state at rest.

Although the fluctuations measured in the BOLD signal during rest are much slower than the $\alpha$ rhythm, there are nevertheless similarities between these resting state fMRI studies and the present study. We find that $\alpha$ power and peak frequency increase as visual input is restricted. It has been well demonstrated that perception and detection of external events is prioritized at certain phases of the $\alpha$ cycle. Therefore, the $\alpha$ rhythm itself may reflect brief fluctuations between interoceptive and exteroceptive states. Under restricted visual input, the power of this rhythm becomes higher but the frequency of these fluctuations increases, allowing the brain to sample the external environment more frequently for adaptive behavior. Additional research using combined EEG and fMRI may help to clarify the relationship between interoceptive and exteroceptive states, the $\alpha$ rhythm, and low frequency BOLD fluctuations.

Analyses on the rates of blinking and eye movements across resting state conditions were conducted to determine compliance with task instructions (i.e., subjects closed their eyes during the ECD condition and maintained fixation in all three conditions). We found no differences in the rate of eye movements across the three resting state conditions, indicating that eye movements were equally minimized in all three conditions. This suggests that the differences found here between light and dark conditions cannot be explained by differences in attention or cognitive control exerted to maintain fixation in the EOF condition, and instead reflect the sensory differences across conditions. We did observe differences in the rate of blinking across conditions. As would be expected, blinking was significantly reduced in the ECD condition compared to both the EOD and EOF conditions, indicating that participants complied with instructions to close their eyes. Interestingly, blinking was also significantly reduced in the EOD condition compared to the EOF condition. Rates of blinking slow with increased task demands and mental engagement, presumably to minimize the risk of missing task-relevant information during eye closure (Drew, 1951; Baumstimler and Parrot, 1971; Stern and Skelly, 1984; Oh et al., 2012). While task demands were not high during these resting state conditions, the reduced rate of blinking in the EOD condition compared to the EOF condition may again reflect a tendency of the visual system to prioritize detection of weak visual input under impoverished visual conditions, consistent with the increase in $\alpha$ peak frequency shown here under conditions of reduced luminance. Alternatively, the higher rates of blinking in the EOF condition compared to the EOD condition may have been a result of the light eliminating goggles used in the EOD condition, which may have produced differences in eye dryness between the two conditions.

Intraindividual shifts in $\alpha$ peak frequency have been noted with changes in cognitive engagement (Haegens et al., 2014), physical task demands (Hülsdünker et al., 2016), and hormone levels (Brötzner et al., 2014). Our results add to this literature and suggest that even changes in sensory input may cause shifts in $\alpha$ peak frequency. We demonstrate across three different resting conditions that visual input modulates both $\alpha$ peak frequency and power. As visual input is reduced, both $\alpha$ peak frequency and power increase. These results have important implications for future studies of the $\alpha$ rhythm. Given the impact of visual input on $\alpha$ peak frequency and power, care should be taken when choosing a resting comparison condition. Visual input in the resting condition should match the task condition as closely as possible, as manipulating the visual input may bias frequency and power in a way that precludes interpretation of task effects.

\section{References}

Adrian ED, Matthews BHC (1934) The Berger rhythm: potential changes from the occipital lobes in man. Brain 57:355-385.

Baumstimler Y, Parrot J (1971) Stimulus generalization and spontaneous blinking in man involved in a voluntary activity. J Exp Psychol 88:95-102.

Bell AJ, Sejnowski TJ (1995) A non-linear information maximisation algorithm that performs blind separation. In: Advances in Neural Information Processing Systems, pp 467-474. Cambridge: MIT Press.

Benedetto A, Lozano-Soldevilla D, VanRullen R (2018) Different responses of spontaneous and stimulus-related alpha activity to ambient luminance changes. Eur J Neurosci 48:2599-2608.

Ben-Simon E, Podlipsky I, Okon-Singer H, Gruberger M, Cvetkovic D, Intrator N, Hendler T (2013) The dark side of the alpha rhythm: fMRI evidence for induced alpha modulation during complete darkness. Eur J Neurosci 37:795-803.

Berger H (1929) Über das elektrenkephalogramm des menschen. Eur Arch Psychiatry Clin Neurosci 87:527-570.

Bianciardi M, Fukunaga M, van Gelderen P, Horovitz SG, de Zwart JA, Duyn JH (2009) Modulation of spontaneous fMRI activity in human visual cortex by behavioral state. Neuroimage 45:160-168.

Boytsova YA, Danko SG (2010) EEG differences between resting states with eyes open and closed in darkness. Hum Physiol 36: 367-369.

Brötzner CP, Klimesch W, Doppelmayr M, Zauner A, Kerschbaum $\mathrm{HH}$ (2014) Resting state alpha frequency is associated with menstrual cycle phase, estradiol and use of oral contraceptives. Brain Res 1577:36-44.

Cecere R, Rees G, Romei V (2015) Individual differences in alpha frequency drive crossmodal illusory perception. Curr Biol 25:231235.

Cohen MX (2014) Fluctuations in oscillation frequency control spike timing and coordinate neural networks. J Neurosci 34:8988-8998.

Cooper NR, Croft RJ, Dominey SJ, Burgess AP, Gruzelier JH (2003) Paradox lost? Exploring the role of alpha oscillations during exter- 
nally vs. internally directed attention and the implications for idling and inhibition hypotheses. Int J Psychophysiol 47:65-74.

Cousineau D (2005) Confidence intervals in within-subject designs: a simpler solution to Loftus and Masson's method. Tutor Quant Methods Psychol 1:42-45.

Drew GC (1951) Variations in reflex blink-rate during visual-motor tasks. Q J Exp Psychol 3:73-88.

Ergenoglu T, Demiralp T, Bayraktaroglu Z, Ergen M, Beydagi $\mathrm{H}$, Uresin Y (2004) Alpha rhythm of the EEG modulates visual detection performance in humans. Cogn Brain Res 20:376-383.

Fox MD, Snyder AZ, Vincent JL, Corbetta M, Van Essen DC, Raichle ME (2005) The human brain is intrinsically organized into dynamic, anticorrelated functional networks. Proc Natl Acad Sci USA 102: 9673-9678.

Fransson P (2005) Spontaneous low-frequency BOLD signal fluctuations: an $\mathrm{fMRI}$ investigation of the resting-state default mode of brain function hypothesis. Hum Brain Mapp 26:15-29.

Gasser T, Bächer P, Steinberg H (1985) Test-retest reliability of spectral parameters of the EEG. Electroencephalogr Clin Neurophysiol 60:312-319.

Grandy TH, Werkle-Bergner M, Chicherio C, Schmiedek F, Lövdén $M$, Lindenberger $U$ (2013) Peak individual alpha frequency qualifies as a stable neurophysiological trait marker in healthy younger and older adults. Psychophysiology 50:570-582.

Greicius MD, Krasnow B, Reiss AL, Menon V (2003) Functional connectivity in the resting brain: a network analysis of the default mode hypothesis. Proc Natl Acad Sci USA 100:253-258.

Gutmann B, Mierau A, Hülsdünker T, Hildebrand C, Przyklenk A, Hollmann W, Strüder HK (2015) Effects of physical exercise on individual resting state EEG alpha peak frequency. Neural Plast 2015:1-6.

Haegens S, Cousijn H, Wallis G, Harrison PJ, Nobre AC (2014) Interand intra-individual variability in alpha peak frequency. Neuroimage 92:46-55.

Hanslmayr S, Aslan A, Staudigl T, Klimesch W, Herrmann CS, Bäuml $\mathrm{K}-\mathrm{H}$ (2007) Prestimulus oscillations predict visual perception performance between and within subjects. Neuroimage 37:14651473.

Hülsdünker T, Mierau A, Strüder HK (2016) Higher balance task demands are associated with an increase in individual alpha peak frequency. Front Hum Neurosci 9:695.

Jaegle A, Ro T (2014) Direct control of visual perception with phasespecific modulation of posterior parietal cortex. J Cogn Neurosci 26:422-432.

Jao T, Vértes PE, Alexander-Bloch AF, Tang I-N, Yu Y-C, Chen J-H, Bullmore ET (2013) Volitional eyes opening perturbs brain dynamics and functional connectivity regardless of light input. Neuroimage 69:21-34.

Kondacs A, Szabó M (1999) Long-term intra-individual variability of the background EEG in normals. Clin Neurophysiol 110:17081716.

Marx E, Stephan T, Nolte A, Deutschländer A, Seelos KC, Dieterich $M$, Brandt T (2003) Eye closure in darkness animates sensory systems. Neuroimage 19:924-934.

Marx E, Deutschländer A, Stephan T, Dieterich M, Wiesmann M, Brandt T (2004) Eyes open and eyes closed as rest conditions: impact on brain activation patterns. Neuroimage 21:1818-1824.
Mathewson KE, Gratton G, Fabiani M, Beck DM, Ro T (2009) To see or not to see: prestimulus phase predicts visual awareness. J Neurosci 29:2725-2732.

McAvoy M, Larson-Prior L, Nolan TS, Vaishnavi SN, Raichle ME, d'Avossa G (2008) Resting states affect spontaneous BOLD oscillations in sensory and paralimbic cortex. J Neurophysiol 100:922931.

Morey RD (2008) Confidence intervals from normalized data: a correction to Cousineau (2005). Tutor Quant Methods Psychol 4:6164.

Oh J, Jeong SY, Jeong J (2012) The timing and temporal patterns of eye blinking are dynamically modulated by attention. Hum Mov Sci 31:1353-1365.

Ray WJ, Cole HW (1985a) EEG alpha activity reflects attentional demands, and beta activity reflects emotional and cognitive processes. Science 228:750-752.

Ray WJ, Cole HW (1985b) EEG activity during cognitive processing: influence of attentional factors. Int J Psychophysiol 3:43-48.

Ro T (2019) Alpha oscillations and feedback processing in visual cortex for conscious perception. J Cogn Neurosci 31:948-960.

Romei V, Brodbeck V, Michel C, Amedi A, Pascual-Leone A, Thut G (2008) Spontaneous fluctuations in posterior $\alpha$-band EEG activity reflect variability in excitability of human visual areas. Cereb Cortex 18:2010-2018.

Salinsky MC, Oken BS, Morehead L (1991) Test-retest reliability in EEG frequency analysis. Electroencephalogr Clin Neurophysiol 79:382-391.

Samaha J, Postle BR (2015) The speed of alpha-band oscillations predicts the temporal resolution of visual perception. Curr Biol 25:2985-2990.

Schupp HT, Lutzenberger W, Birbaumer N, Miltner W, Braun C (1994) Neurophysiological differences between perception and imagery. Cogn Brain Res 2:77-86.

Stanley J (1971) Reliability. In: Educational measurement (Thorndike RL, ed). Washington, DC: American Council on Education.

Stern JA, Skelly JJ (1984) The eye blink and workload considerations. Proc Hum Factors Soc Annu Meet 28:942-944.

Thut G, Schyns PG, Gross J (2011) Entrainment of perceptually relevant brain oscillations by non-invasive rhythmic stimulation of the human brain. Front Psychol 2:170.

Worden MS, Foxe JJ, Wang N, Simpson GV (2000) Anticipatory biasing of visuospatial attention indexed by retinotopically specific-band electroencephalography increases over occipital cortex. J Neurosci 20:1-6.

Xu P, Huang R, Wang J, Van Dam NT, Xie T, Dong Z, Chen C, Gu R, Zang Y-F, He Y, Fan J, Luo Y (2014) Different topological organization of human brain functional networks with eyes open versus eyes closed. Neuroimage 90:246-255.

Yan C, Liu D, He Y, Zou Q, Zhu C, Zuo X, Long X, Zang Y (2009) Spontaneous brain activity in the default mode network is sensitive to different resting-state conditions with limited cognitive load. PLoS One 4:e5743.

Zou Q, Long X, Zuo X, Yan C, Zhu C, Yang Y, Liu D, He Y, Zang Y (2009) Functional connectivity between the thalamus and visual cortex under eyes closed and eyes open conditions: a restingstate fMRI study. Hum Brain Mapp 30:3066-3078. 